\title{
Clear Cell Carcinoma of the Endometrium:
}

\section{Evaluation of Prognostic Parameters in a Multi-institutional Cohort of 165 Cases}

\author{
Eman Abdulfatah, MD*, Sharif Sakr, MD*, Sumi Thomas, MD $^{\dagger}$, Zaid Al-Wahab, MD, David G. \\ Mutch, MD ${ }^{\ddagger}$, Sean Dowdy, MD $^{\dagger}$, Sudeshna Bandyopadhyay, MD ${ }^{*}$, Adnan Munkarah, MD \\ Mohamed Elshaikh, MD", Robert Morris, MD", and Rouba Ali-Fehmi, MD* \\ *Department of Pathology, Wayne State University, Detroit, Ml; \\ tDepartment of Obstetrics and Gynecology, Mayo Clinic, Rochester, MN; \\ ‡Department of Obstetrics and Gynecology, Washington University, St Louis, MO; \\ $\S$ Department of Obstetrics and Gynecologym, Washington University, St Louis, MO \\ "Department of Radiation Oncology, Henry Ford Hospital/Wayne State University; \\ IDepartment of Obstetrics and Gynecology, Wayne State University, Detroit, MI.
}

\section{Abstract}

Objectives: Clear cell carcinoma (CCC) comprises a rare yet an aggressive subtype, accounting for less than 5\% of all uterine carcinomas. Several clinicopathologic features have been predictive of poor prognosis; however, data remain controversial. The aim of this study was to evaluate the clinicopathologic features of a multi-institutional cohort of endometrial CCC in order to identify which, if any, have prognostic significance.

Methods: Retrospective review of endometrial CCC diagnosed between 1995 and 2012 at 3 institutions was conducted to evaluate clinicopathologic parameters: age, race, tumor size, stage, myometrial invasion (MI), lymphovascular invasion, lymph node and adnexal involvement, adjuvant therapy, and outcomes. Data were analyzed using Fisher exact, Cox regression, and Kaplan-Meier analyses.

Results: Patients' ages ranged from 36 to 90 years (median, 67 years). The median tumor size was $3.6 \mathrm{~cm}$. Inner-half MI was present in 44\%, lymphovascular invasion in 34\%, adnexal involvement in $16 \%$, and lymph node metastasis in $30 \%$ of cases. Fifty-eight percent of the patients presented with early-stage disease. The 5-year overall survival (OS) was 58\%. Shorter disease-free interval (DFI) was significantly associated with older age at diagnosis ( $>70$ years), advanced-stage disease, adnexal involvement, and deep MI $(P=0.005, P=0.001, P=0.001$, and $P$ $=0.003$, respectively). Patients who received adjuvant chemotherapy had a significantly worse DFI and 5-year OS ( $P=0.001$ and $P=0.001$, respectively). A significantly shorter 5-year OS was noted with advanced stage (IIIYIV) and presence of adnexal involvement $(P=0.001$ and $P=$ 0.021 , respectively). On Cox regression analysis, advanced-stage disease, older age, and adnexal

Address correspondence and reprint requests to Eman Abdulfatah, MD, Department of Pathology, Wayne State University, 540 E Canfield Ave, Detroit, MI 48201. eabdulfa@med.wayne.edu; Eman.abdulfatah@gmail.com. 
involvement were significant independent predictors of worse DFI $(P=0.001, P=0.005$, and $P=$ 0.019 , respectively), whereas inner-half MI was a significant independent predictor of longer DFI $(P=0.004)$. Adjuvant radiotherapy alonewas a significant independent predictor of better 5 -year OS $(P=0.012)$.

Conclusions: In our series of endometrial CCC, older age at diagnosis, advanced stage, deep MI, and adnexal involvement were independent poor prognostic factors. Adjuvant radiotherapy had a significant positive impact on 5-year OS.

\section{Keywords}

Clear cell carcinoma; Clinicopathologic; Prognostic; Radiotherapy

Endometrial carcinoma (EC) is the most common gyneco-logic malignancy in the United States, with 60,050 new cases and 10,470 deaths estimated in $2015 .{ }^{1}$ The dualistic model established on morphologic basis more than 20 years ago divides EC into 2 broad subtypes, defined as type I and type II. Type I, which encompasses approximately $80 \%$ to $85 \%$ of the cases, is estrogen dependent and consists of endometrioid carcinoma and its histologic variants; type II endometrial cancer, on the other hand, is estrogen independent and includes serous carcinoma, clear cell carcinoma (CCC), and carcinosarcoma. ${ }^{2-4} \mathrm{~A}$ decade later, different molecular profiles have been described for each category with PTEN gene silencing, DNA mismatch repair genes defects, and KRAS and PIK3CA gene mutations being the major alterations in type I, whereas type II tumors harbor p53 gene mutations, p16 gene inactivation, Her-2/neu overexpression, and loss of heterozygosity on several chromosomes. ${ }^{5-8}$ Recently, 4 prognostically significant molecular subtypes of EC were recognized by The Cancer Genome Atlas group based on a combination of somatic nucleotide substitutions, microsatellite instability, and somatic copy number alterations, namely, ultramutated (POLE) group, microsatellite instability, copy-number low, and copynumber high. ${ }^{9}$

Clear cell carcinoma comprises a rare yet an aggressive subtype, accounting for less than 5\% of all uterine carcinomas. ${ }^{10}$ Microscopically, CCC is characterized by clear, often eosinophilic, hobnail cells that exhibit different architectural patterns including solid, papillary, and tubulocystic. Similar histologic features are displayed when CCC develops in the ovary, cervix, and vagina. ${ }^{11}$ In contrast to endometrioid and serous carcinomas, limited data are available regarding the potential precursor lesions for $\mathrm{CCC}$ of the endometrium. Recent studies identified a spectrum of atypical glandular changes (isolated glands or surface epithelium that displayed cytoplasmic clarity and/or eosinophilia with varying degrees of nuclear atypia) within the endometrium adjacent to $\mathrm{CCC}$, suggesting that these lesions could represent the earliest morphologic features of this tumor. ${ }^{12}$ Patients with CCC tend to be older, are more likely to present at higher-stage disease, and show a worse prognosis when compared with endometrioid carcinoma. ${ }^{13-15}$ A 5-year overall survival (OS) rate of $42.3 \%$ to $62.5 \%$ has been reported in advanced-stage (stages IIIYIV) disease, ${ }^{16,17}$ and hence more aggressive adjuvant treatment is recommended. ${ }^{18}$

To date, limited information is reported in the literature regarding the prognostic parameters of CCC. Several clinicopathologic features including age older than 65 years, extra-uterine 
disease, advanced stage, and presence of lymph node metastasis have been predictive of poor prognosis; however, data remain controversial. ${ }^{19}$ The aim of this study was to evaluate the clinicopathologic features of a large multi-institutional cohort of endometrial CCC in order to identify which, if any, have prognostic significance.

\section{MATERIALS AND METHODS}

After obtaining institutional review board approval, a retrospective review of endometrial CCC $(n=165)$ diagnosed between 1995 and 2012 at 3 institutions (Karmanos Cancer Institute/Wayne State University, Washington University, and Mayo Clinic) was conducted. Only patients who underwent definite surgical treatment were included. Electronic medical records and pathology reports were reviewed to analyze clinical parameters (age at diagnosis, adjuvant treatment, lymph node sampling, and sites of recurrence), pathological variables (tumor size and stage), and survival data (vital status, disease-free interval [DFI], and OS). In addition, we separately analyzed parameters that are part of the staging system (depth of myometrial invasion [MI], lymphovascular invasion [LVI], lymph node, and adnexal involvement) and are of prognostic significance individually. Tumor stagewas assigned accordingtotheInternational Federation of Gynecology and Obstetrics (FIGO) criteria. ${ }^{20}$ Stages I-II were considered as early stage, whereas stages III-IV tumors were defined as advanced-stage disease. Representative hematoxylin-eosin-stained whole-tissue sections of a subset of cases were retrieved and reviewed by 2 gynecologic pathologists (R.A.F. and S.B.) to confirm the diagnosis as per the World Health Organization criteria. Mixed carcinomas defined as carcinomas with 1 or more other components comprising at least $10 \%$ of the tumor volume were excluded. Endometrioid carcinomas exhibiting clear cell features were also excluded. To confirm the presence of LVI, a subset of cases $(n=65)$ were stained with CD34 immunostain. Immunohistochemical staining was performed on samples fixed in $10 \%$ neutral-buffered formalin and embedded in paraffin. Slides were blocked for 30 minutes. Monoclonal mouse anti-human CD34 (clone: QBEnd 10; DAKO, Glostrup, Denmark) was used as the primary antibody (dilution 1:200). Slides were incubated at room temperature for 30 minutes. Detection of antibody binding was obtained using Optiview (Ventana) after a polymer-based amplificationstep(Ultraview;Ventana).Slideswerethencounterstained with hematoxylin and cover slipped. Only cases with membranous staining were considered to be positive.

Long-term follow-up data were censored at the date of last contact. Disease-free interval was calculated from the date of diagnosis until date of recurrence, death, or last follow-up. Overall survival was calculated from the date of diagnosis until death or date of last followup. Statistical analyses were conducted using Fisher exact (2-tailed) $\mathrm{W} x^{2}$ Student $t$ test, and log-rank tests. Univariate Cox regression analysis was performed to evaluate the prognostic impact of various clinicopathologic factors on DFI and OS. Multivariate Cox regression analysis was performed according to the variables that showed significance on univariate analysis. The hazard ratios (HRs) were estimated with $95 \%$ confidence interval (CI). Kaplan-Meier graphs were used to illustrate both OS and DFI. Overall survival was analyzed at 5-year interval because the study was multi-institutional with different treatment protocols, as well as the lack of long periods of follow-up for some of the cases. All analyses were performed with SPSS software, and statistical significance was set at $\mathrm{P}<0.05$. 


\section{RESULTS}

\section{Clinicopathologic Features}

A total of 165 cases with confirmed diagnosis of endometrial CCC were included in our study. The clinicopathologic features of these patients and their tumors are detailed in Table 1. Representative histologic features are shown in Figure 1. The median age at diagnosis was 67 years (mean, 66 years; range, 36-90 years). The median tumor size at the time of surgical resection was $3.6 \mathrm{~cm}$ (mean, $4.1 \mathrm{~cm}$; range, $0.1-17.0 \mathrm{~cm}$ ), with the majority of the tumors measuring more than $2.0 \mathrm{~cm}$ in greatest dimension (80\%). Myometrial invasion extending to the inner half was present in a high proportion of cases (70/165 [44\%]), whereas slightly fewer cases had outer half involvement (52/165 [32\%]), and no invasion was seen in 24\% (38/ 165) of cases. Lymphovascular invasion was identified in 34\% (55/165), whereas adnexal involvement was seen in 16\% (26/ 165) of cases. Most patients (135/165 [82\%]) had lymphadenectomy performed as part of surgical staging, and positive lymph node metastasis was documented in $30 \%$ (40/135) of cases. The FIGO stage distribution was $42 \%$ stage I, $16 \%$ stage II, $24 \%$ stage III, and $18 \%$ stage IV. Ninety-five patients (58\%) presented with early-stage disease.

\section{Treatment Characteristics}

All patients underwent total hysterectomy and bilateral salpingo-oophorectomy.

Lymphadenectomy was performed in $82 \%$ (135/165), whereas omentectomy was performed in only $52 \%$ (86/165) of patients. Radiotherapy in the form of vaginal brachytherapy and/or whole pelvic radiation therapy was part of the adjuvant treatment in $48 \%$ (78/165) of patients. Fifty-six patients (34\%) received adjuvant chemotherapy. Of these, 90\% (50/56) were treated with a platinum-based regimen (carboplatin and paclitaxel).

\section{Survival Analysis}

The median follow-up period was 29 months (range, 1-267 months). During the follow-up interval, tumor recurrence was observed in 61 (37\%) of cases. The median time to tumor recurrence (DFI) was 64 months (range, 1-188 months). The 5-year OS was 58\%.

Recurrence was significantly associated with tumors deeply invading into the myometrium, advanced-stage (stages III-IV) disease, and adnexal involvement and was higher in patients who received adjuvant chemotherapy only $(P=0.049, P=0.001, P=0.001$, and $P=0.040$, respectively) (Table 2). Patients with recurrent tumors had a significantly worse 5-year OS $(17 \%$ vs $83 \%, P=0.001)$.

Kaplan-Meier analysis for DFI (Fig. 2) and 5-year OS (Fig. 3) was performed according to the variables that showed significance on univariate analysis. Shorter DFI was significantly associatedwitholderageat diagnosis(>70years), advanced-stage disease, adnexal involvement, and deep MI ( $P=0.005, P=0.001, P=0.001$, and $P=0.003$, respectively). Patients who received adjuvant chemotherapy had a significantly worse DFI and 5-year OS ( $P=0.001$ and $P=0.001$, respectively). A significantly shorter 5-year OS was noted with advanced stage (III-IV) and presence of adnexal involvement $(P=0.001$ and $P=0.021$, respectively). 
Cox regression analyses for the associations between clinicopathologic features, DFI, and 5year OS are presented in Tables 3 and 4 . Advanced-stage disease, older age at diagnosis, and adnexal involvement were significant independent predictors of worse DFI $(P=0.001, P=$ 0.005 , and $P=0.019$, respectively), whereas inner-half MI was a significant independent predictor of a longer DFI (HR, 0.35; CI, 0.14-0.69; $P=0.004)$. Adjuvant radiotherapy alone was a significant independent predictor of better 5-year OS (HR, 0.56; CI, 0.35Y0.87; $P=$ $0.012)$.

\section{DISCUSSION}

Uterine CCC is distinctly uncommon, comprising less than $5 \%$ of all ECs. ${ }^{21}$ In contrast to their cervical and vaginal counterparts, no association with diethylstilbestrol has been described; however, radiation and tamoxifen have been implicated in a subset of these tumors. ${ }^{22}$ Patients are usually post-menopausal and tend to be older than those with endometrioid carcinoma, presenting at a median age of 66 to 68 years. ${ }^{10,13}$ Despite its designation as a high-grade carcinoma, disease is confined to the uterus (stages I-II) in the majority of the cases. Myometrial invasion is present in approximately $80 \%$ of cases and LVI in approximately $25 \% .{ }^{16}$ In concordance with these findings, Abeler et $\mathrm{al}^{23}$ published a series of 181 patients with CCC who presented predominately with early-stage disease with documented MI and LVI in 75\% and 37\%, respectively. Pathologic stage and patient age were believed to be the most important prognostic factors of CCC based on a study by Hamilton et al, ${ }^{10}$ who conducted one of the largest studies to date on SEER (Surveillance, Epidemiology and End Results) data comparing the clinicopathologic prognostic factors and outcomes of patients with serous carcinomas, CCC, and high- grade endometrioid carcinomas (G3EC). In their series, patients diagnosed as having serous carcinomas and CCC were significantly older (median age, 70 years and 68 vs 66 years, respectively) and had a significantly poorer prognosis compared with those with G3EC. It has been also noted by previous reviews that CCC has a propensity for extrauterine disease spread and that relapses occur more commonly beyond the pelvis. ${ }^{24}$ Similarly, in our series, the majority of the patients presented at an older age (median age of 67 years), with stage I-II in 58\%, MI in $76 \%$, and LVI in 34\%. Furthermore, advanced stage, older age at diagnosis, presence of deep MI, and adnexal involvement were significant predictors of worse prognosis, confirming the previous literature. Consistent with our results and the previously published data, a recent study by Varughese et $\mathrm{al}^{25}$ showed that age and stage have a significant impact on OS. In addition, they reported that LVI was found to have no effect on survival in CCC.

Lymphovascular invasion is an essential step in the process of tumor metastases and is an important prognostic factor in EC. Currently, the standard method for assessing LVI is by morphologic examination of hematoxylin-eosin-stained sections. Strict criteria for LVI based on morphology include tumor clusters attached to the vessel wall, endothelialization of the tumor clusters, or the presence of a thrombus connecting the tumor clusters to the vessel wall. Alexander-Sefre et $\mathrm{al}^{26}$ in vestigated the clinical significance of LVI detected by immunohistochemical staining for vascular endothelial markers in stage I endometrioid EC patients and concluded that immunohistochemically detected LVI appears to be of no statistically significant clinical value. In an attempt to validate these findings in CCC, we studied the impact of LVI on prognosis following the confirmation of its presence by CD34 
immuno-stain. However, we failed to demonstrate any significant correlation with DFI and OS, supporting the previous publications. Conversely, Abeler and Kjørstad ${ }^{16}$ observed that LVI and MI are powerful prognosticators in CCC. It has been also well established that patients with CCC have a greater risk of thromboembolic events particularly at higher-stage disease; however, the exact mechanism underlying this phenomenon is not well known. ${ }^{26,27}$ Future studies to further investigate this hypothesis are recommended, as well as considerations for extended prophylaxis in these patients to prevent such events.

While the standard first-line therapy for uterine CCC is total abdominal hysterectomy and bilateral salpingo-oophorectomy with comprehensive surgical staging (peritoneal washing, omentectomy, and pelvic and para-aortic lymphadenectomy), optimal postoperative management remains to be fully defined. Treatment strategies vary at different stages, and given the small number of women affected with uterine $\mathrm{CCC}$, factors associated with improved survival are difficult to discern. The Society of Gynecologic Oncology reported that adjuvant pelvic and/or whole abdominal radiotherapy have not been shown to be beneficial in women diagnosed as having uterine CCC. In stage III or IV (extrauterine disease), as well as in women with recurrent disease, they recommend the use of adjuvant chemotherapy with cisplatin, taxol, and doxorubicin either in doublet or triplet combination. ${ }^{18}$ Conversely, an observational study of 80 patients with CCC demonstrated that vaginal brachytherapy, whether alone or in combination with other radiation therapy, had a significant impact on OS (median, 140 vs 50 months; $P=0.02$ ) but no improvement in progression-free survival. It was also noted that adjuvant chemotherapy alone or in addition to vaginal brachytherapy had no significant impact on OS and progression-free survival. ${ }^{25} \mathrm{In}$ agreement with these results, our study showed that patients who received radiation therapy had significantly better 5-year OS, whereas patients who received both radiotherapy and chemotherapy or chemotherapy alone had no improvement in their outcomes.

In a multi-institutional review of surgical management and adjuvant therapy for uterine CCC, the authors concluded that vaginal brachytherapy may be adequate for stages I and II confirmed by the fact that lymph node dissection and pelvic radiation therapy appear to be of benefit for patients at risk of lymph node recurrence. ${ }^{27}$ Smith et al ${ }^{15}$ assessed 26 patients with stages I-IV CCC of the endometrium who received postoperative irradiation. The 3year disease-free survival and OS were $47 \%$ and $68 \%$ for the entire cohort, $87 \%$ and $87 \%$ for patients with stages I and II disease, and $32 \%$ and $61 \%$ for those with stages III and IV disease, respectively. Accordingly, adjuvant radiotherapy was an effective treatment for patients with early-stage disease but not for those with advanced-stage tumors for whom clinical trial of radiotherapy with concurrent or sequential chemotherapy was recommended. These 2 studies, in addition to our study, seem to contradict the findings of the Taiwanese Gynecologic Oncology Group study, which showed that adjuvant chemotherapy provided a better 5 year progression-free survival and OS than radiotherapy in early-stage uterine CCC. 27

Although we note the limitations of our study, namely, a relatively small sample size and a retrospective review, it is one of the largest series to date addressing uterine CCC. In addition, data were obtained by the collaboration of 3 major academic institutions offering a diverse patient population and similar patterns of practice and the ability to access patient 
details and expert pathology review. Ideally, prospective analysis of large- scale clinical trials shall provide insight into the most effective postoperative treatment modalities.

In conclusion, endometrial CCC was found to be more common in older women and was noted to have worse prognosis with advanced stage, deep MI, adnexal involvement, and older age at diagnosis. Adjuvant radiotherapy had a significant positive impact on 5-year OS, hence suggesting that more aggressive adjuvant treatment is recommended. Future research at the molecular level might shed more light on the biology of this tumor.

\section{REFERENCES}

1. Siegel RL, Miller KD, Jemal A. Cancer statistics, 2016. CA Cancer J Clin. 2016;66:7-30. [PubMed: 26742998]

2. Bokhman JV. Two pathogenetic types of endometrial carcinoma. Gynecol Oncol. 1983;15:10-17. [PubMed: 6822361]

3. Prat J Prognostic parameters of endometrial carcinoma. Hum Pathol. 2004;35:649-662. [PubMed: 15188130]

4. Prat J, Gallardo A, Cuatrecasas M, et al. Endometrial carcinoma: pathology and genetics. Pathology. 2007;39:72-87. [PubMed: 17365824]

5. Doll A, Abal M, Rigau M, et al. Novel molecular profiles of endometrial cancerVnew light through old windows. J Steroid Biochem Mol Biol. 2008;108:221-229. [PubMed: 18061438]

6. Santin AD, Bellone S, Van Stedum S, et al. Amplification of c-erbB2 oncogene: a major prognostic indicator in uterine serous papillary carcinoma. Cancer. 2005;104:1391-1397. [PubMed: 16116605]

7. Catasus L, Gallardo A, Cuatrecasas M, et al. PIK3CA mutations in the kinase domain (exon 20) of uterine endometrial adenocarcinomas are associated with adverse prognostic parameters. Mod Pathol. 2008;21:131-139. [PubMed: 18084252]

8. Bansal N, Yendluri V, Wenham RM. The molecular biology of endometrial cancers and the implications for pathogenesis, classification, and targeted therapies. Cancer Control. 2009;16:8-13. [PubMed: 19078924]

9. Talhouk A, McConechy MK, Leung S, et al. A clinically applicable molecular-based classification for endometrial cancers. Br J Cancer. 2015;113:299-310. [PubMed: 26172027]

10. Hamilton CA, Cheung MK, Osann K, et al. Uterine papillary serous and clear cell carcinomas predict for poorer survival compared to grade 3 endometrioid corpus cancers. Br J Cancer. 2006;94:642-646. [PubMed: 16495918]

11. Matias-Guiu X, Lerma E, Prat J. Clear cell tumors of the female genital tract. Semin Diagn Pathol. 1997;14:233-239. [PubMed: 9383823]

12. Fadare O, Liang SX, Ulukus EC, et al. Precursors of endometrial clear cell carcinoma. Am J Surg Pathol. 2006;30:1519-1530. [PubMed: 17122507]

13. Christopherson WM, Alberhasky RC, Connelly PJ. Carcinoma of the endometrium: I. A clinicopathologic study of clear-cell carcinoma and secretory carcinoma. Cancer. 1982;49:15111523. [PubMed: 7066860]

14. Cirisano FD, Jr, Robboy SJ, Dodge RK, et al. The outcome of stage IYII clinically and surgically staged papillary serous and clear cell endometrial cancers when compared with endometrioid carcinoma. Gynecol Oncol. 2000;77:55-65. [PubMed: 10739691]

15. Smith RS, Kapp DS, Chen Q, et al. Treatment of high-risk uterine cancer with whole abdominopelvic radiation therapy. Int J Radiat Oncol Biol Phys. 2000;48:767-778. [PubMed: 11020574]

16. Abeler VM, Kjørstad KE. Clear cell carcinoma of the endometrium: a histopathological and clinical study of 97 cases. Gynecol Oncol. 1991;40:207-217. [PubMed: 2013441]

17. Creasman WT, Odicino F, Maisonneuve P, et al. Carcinoma of the corpus uteri. FIGO 26th annual report on the results of treatment in gynecological cancer. Int J Gynaecol Obstet. 2006;95(suppl 1):S105-S143. 
18. Olawaiye AB, Boruta DM 2nd. Management of women with clear cell endometrial cancer: a Society of Gynecologic Oncology (SGO) review. Gynecol Oncol. 2009;113:277-283. [PubMed: 19251307]

19. Fadare O, Zheng W, Crispens MA, et al. Morphologic and other clinicopathologic features of endometrial clear cell carcinoma: a comprehensive analysis of 50 rigorously classified cases. Am J Cancer Res. 2013;3:70-95. [PubMed: 23359866]

20. Pecorelli S, Zigliani L, Odicino F. Revised FIGO staging for carcinoma of the cervix. Int J Gynaecol Obstet. 2009;105:107-108. [PubMed: 19342051]

21. Cirisano FD, Jr, Robboy SJ, Dodge RK, et al. Epidemiologic and surgicopathologic findings of papillary serous and clear cell endometrial cancers when compared to endometrioid carcinoma. Gynecol Oncol. 1999;74:385-394. [PubMed: 10479498]

22. Dallenbach-Hellweg G, Hahn U. Mucinous and clear cell adenocarcinomas of the endometrium in patients receiving antiestrogens (tamoxifen) and gestagens. Int J Gynecol Pathol. 1995;14:7-15. [PubMed: 7883429]

23. Abeler VM, Vergote IB, Kjørstad KE, et al. Clear cell carcinoma of the endometrium. Prognosis and metastatic pattern. Cancer. 1996;78:1740-1747. [PubMed: 8859187]

24. Gadducci A, Cosio S, Spirito N, et al. Clear cell carcinoma of the endometrium: a biological and clinical enigma. Anticancer Res. 2010;30:1327-1334. [PubMed: 20530448]

25. Varughese J, Hui P, Lu L, et al. Clear cell cancer of the uterine corpus: the association of clinicopathologic parameters and treatment on disease progression. J Oncol. 2011;2011: 628084 [PubMed: 22187554]

26. Alexander-Sefre F, Singh N, Ayhan A, et al. Clinical value of immunohistochemically detected lymphovascular invasion in endometrioid endometrial cancer. Gynecol Oncol. 2004;92:653-659. [PubMed: 14766262]

27. Kerr R, Stirling D, Ludlam CA. Interleukin 6 and haemostasis. Br J Haematol. 2001;115:3-12. [PubMed: 11722403] 

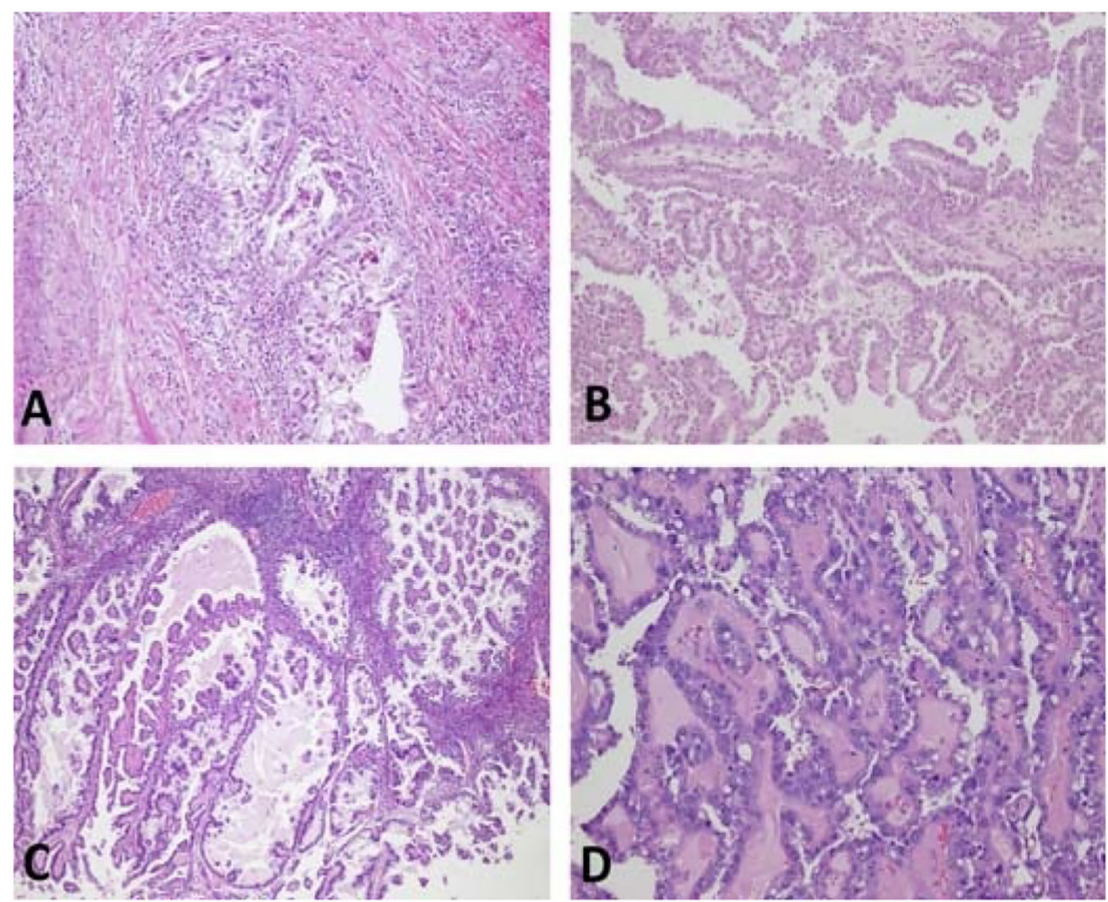

FIGURE 1.

Hematoxylin-eosin photographs of representative slides of cases in our study. A, Clear cell carcinoma within fibromatous background (original magnification $\times 100$ ). B and C, Papillary architecture (original magnification $\times 40$ ). D, Hyalinized cores (original magnification $\times 100$ ). 

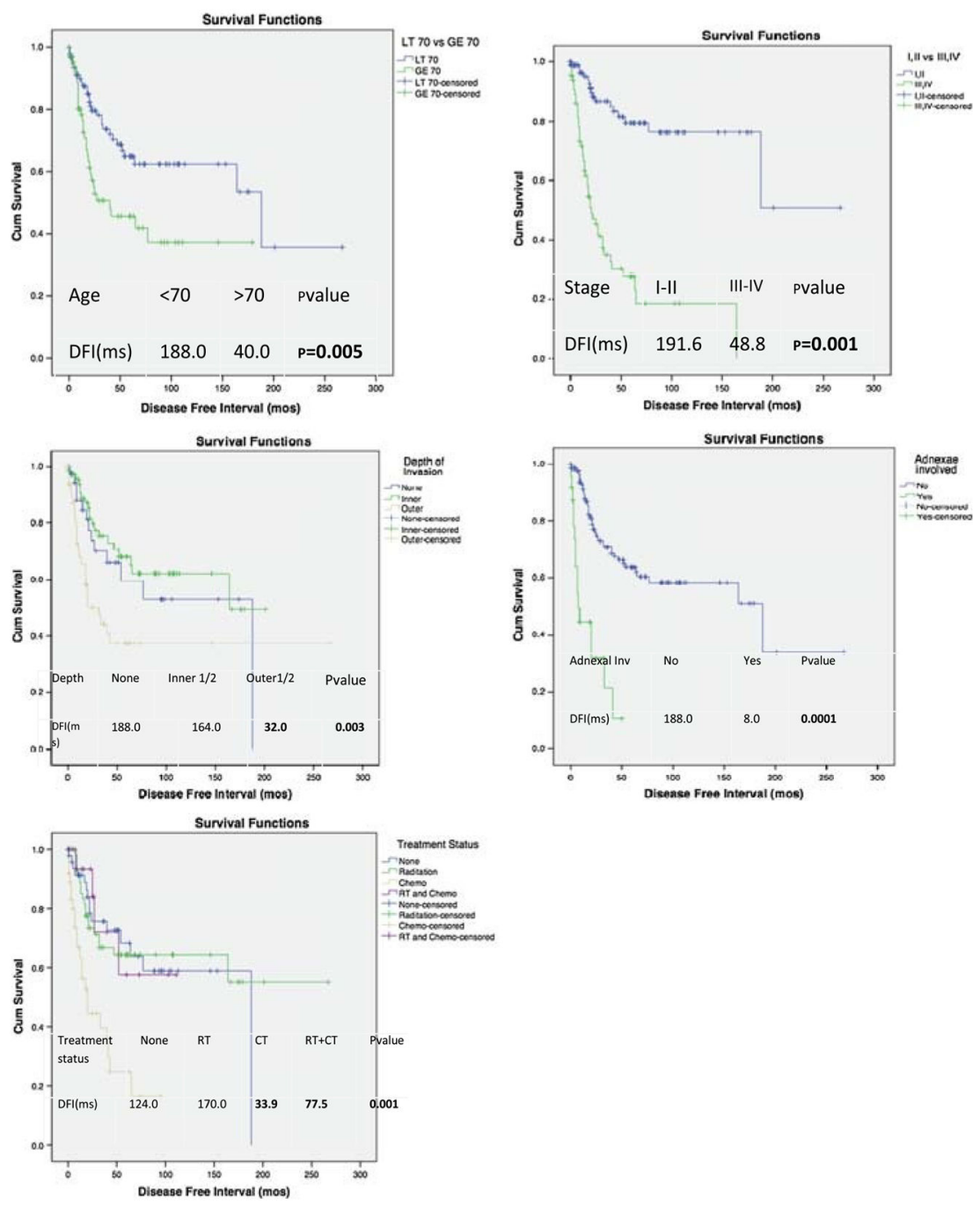

FIGURE 2.

Kaplan-Meier plots of DFI to first recurrence according to age, tumor stage, depth of MI, adnexal involvement, and treatment status. 

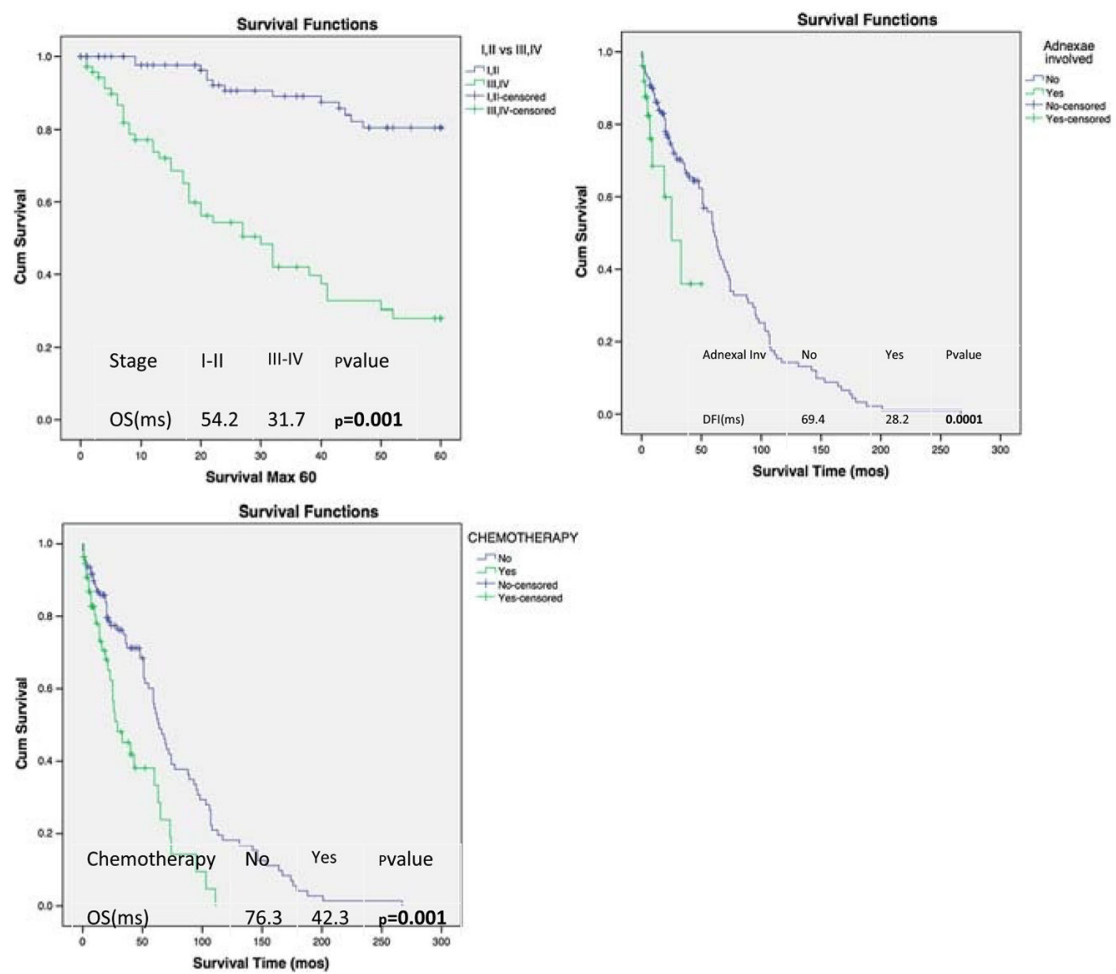

FIGURE 3.

Kaplan-Meier plots of 5-year OS according to significant variables on univariate analysis. 
TABLE 1.

Clinicopathologic characteristics of CCC of endometrium $(n=165)$

\begin{tabular}{|c|c|}
\hline & $\mathrm{n}(\%)^{*}$ \\
\hline Median age (range), y & $67(36-90)$ \\
\hline$<50$ & $13(8)$ \\
\hline $50-70$ & $89(54)$ \\
\hline$>70$ & $63(38)$ \\
\hline Median tumor size (range), $\mathrm{cm}$ & $3.6(0.1-17)$ \\
\hline \multicolumn{2}{|l|}{ Tumor size } \\
\hline$<2 \mathrm{~cm}$ & $22(20)$ \\
\hline$\geq 2 \mathrm{~cm}$ & $86(80)$ \\
\hline \multicolumn{2}{|l|}{ Myometrium invasion } \\
\hline Absent & $38(24)$ \\
\hline Inner half & $70(44)$ \\
\hline Outer half & $52(32)$ \\
\hline \multicolumn{2}{|l|}{ LV1 } \\
\hline Absent & $107(66)$ \\
\hline Present & $55(34)$ \\
\hline \multicolumn{2}{|l|}{ Adnexal metastasis } \\
\hline Absent & $139(84)$ \\
\hline Present & $26(16)$ \\
\hline \multicolumn{2}{|l|}{ LN metastasis $*$} \\
\hline Absent & $95(70)$ \\
\hline Present & $40(30)$ \\
\hline \multicolumn{2}{|l|}{ FIGO stage } \\
\hline I & $69(42)$ \\
\hline II & $26(16)$ \\
\hline III & $40(24)$ \\
\hline IV & $30(18)$ \\
\hline
\end{tabular}


TABLE 2.

Association between clinicopathologic characteristics of CCC and recurrence status

\begin{tabular}{|c|c|c|c|c|}
\hline Parameter & Variable & $\begin{array}{l}\text { Nonrecurrent, } \mathbf{n}(\%) \\
\qquad \mathbf{n}=101\end{array}$ & $\begin{array}{c}\text { Recurrent, } \mathbf{n}(\%)^{*} \\
\mathbf{n}=61\end{array}$ & $P$ \\
\hline \multirow[t]{2}{*}{ Age at diagnosis } & $\leq 70$ & $35(35)$ & $13(21)$ & 0.078 \\
\hline & $>70$ & $66(65)$ & $48(79)$ & \\
\hline \multirow[t]{2}{*}{ Race } & EA & $67(70)$ & $32(70)$ & 1.000 \\
\hline & AA & $29(30)$ & $14(30)$ & \\
\hline \multirow[t]{2}{*}{ Tumor size, $\mathrm{cm}$} & $<2$ & $13(21)$ & $9(20.5)$ & 1.000 \\
\hline & $\geq 2$ & $49(79)$ & $35(79.5)$ & \\
\hline \multirow[t]{3}{*}{ Depth of MI } & Outer half & $25(25.5)$ & $26(44)$ & 0.049 \\
\hline & Inner half & $48(49)$ & $20(34)$ & \\
\hline & None & $25(25.5)$ & $13(22)$ & \\
\hline \multirow[t]{2}{*}{ Stage } & I-II & 78 (77) & $16(26)$ & 0.001 \\
\hline & III-IV & $23(33)$ & $45(74)$ & \\
\hline \multirow[t]{2}{*}{ Lymph node status } & Positive & $30(30)$ & $24(41)$ & 0.169 \\
\hline & Negative & $71(70)$ & $35(59)$ & \\
\hline \multirow[t]{2}{*}{ LVI } & Present & $30(30)$ & $24(41)$ & 0.169 \\
\hline & Absent & $71(70)$ & $35(59)$ & \\
\hline \multirow[t]{2}{*}{ Adnexal involvement } & Present & $8(8)$ & $17(28)$ & 0.001 \\
\hline & Absent & $93(92)$ & $44(72)$ & \\
\hline \multirow[t]{2}{*}{ Omentectomy } & Performed & $53(52.5)$ & $31(51)$ & 0.872 \\
\hline & Not performed & $48(47.5)$ & $40(49)$ & \\
\hline \multirow[t]{2}{*}{ Adjuvant radiotherapy } & Yes & $52(51.5)$ & $24(39)$ & 0.147 \\
\hline & No & $49(48.5)$ & $37(61)$ & \\
\hline \multirow[t]{2}{*}{ Adjuvant chemotherapy } & Yes & $28(28)$ & $27(44)$ & 0.040 \\
\hline & No & $73(72)$ & $34(56)$ & \\
\hline \multirow[t]{2}{*}{ Status at $5 \mathrm{y}$} & Alive & $92(91.1)$ & $18(29.5)$ & 0.0005 \\
\hline & Dead & $9(8.9)$ & $43(70.5)$ & \\
\hline
\end{tabular}

Data in bold means statistically significant.

Data in some cases are missing. 
TABLE 3.

Cox regression analysis for the association of clinicopathologic factors with DFI

\begin{tabular}{|c|c|c|}
\hline Variable & DFI HR $(95 \%$ CI $)$ & $\boldsymbol{P}$ \\
\hline Age at diagnosis & $2.20(1.27-3.82)$ & 0.005 \\
\hline \multicolumn{3}{|l|}{$\leq 70$ vs $>70$} \\
\hline Tumor stage & $7.09(3.18-15.82)$ & 0.001 \\
\hline \multicolumn{3}{|l|}{ I-II vs III-IV } \\
\hline Depth of MI & $0.35(0.14-0.69)$ & $\mathbf{0 . 0 0 4}$ \\
\hline \multicolumn{3}{|l|}{ Inner vs outer half } \\
\hline Adnexal involvement & $2.23(1.14-4.34)$ & 0.019 \\
\hline \multicolumn{3}{|l|}{ Present vs absent } \\
\hline Adjuvant radiotherapy & $0.72(0.32-1.61)$ & 0.425 \\
\hline \multicolumn{3}{|l|}{ Yes vs no } \\
\hline Adjuvant chemotherapy & $1.10(0.48-2.50)$ & 0.815 \\
\hline \multicolumn{3}{|l|}{ Yes vs no } \\
\hline Adjuvant and radiotherapy chemotherapy & $0.71(0.21-2.35)$ & 0.575 \\
\hline Yes vs no & & \\
\hline
\end{tabular}


TABLE 4.

Cox regression analysis for the association of clinicopathologic factors with 5-year OS

\begin{tabular}{lcc}
\hline Variable & 5-y OS HR (95\% CI) & $\boldsymbol{P}$ \\
\hline $\begin{array}{l}\text { Adnexal involvement } \\
\quad \text { Present vs absent }\end{array}$ & $1.68(0.75-3.74)$ & 0.203 \\
$\begin{array}{l}\text { Adjuvant radiotherapy } \\
\quad \text { Yes vs no }\end{array}$ & $0.56(0.35-0.87)$ & $\mathbf{0 . 0 1 2}$ \\
$\begin{array}{l}\text { Adjuvant chemotherapy } \\
\quad \text { Yes vs no }\end{array}$ & $1.46(0.79-2.69)$ & 0.229 \\
$\begin{array}{l}\text { Adjuvant chemotherapy and radiotherapy } \\
\quad \text { Yes vs no }\end{array}$ & $1.49(0.81-2.76)$ & 0.202 \\
\hline
\end{tabular}

